\title{
Erratum to: The Development of Translational Biomarkers as a Tool for Improving the Understanding, Diagnosis and Treatment of Chronic Neuropathic Pain
}

\author{
David A. Buckley ${ }^{1}$ - Elaine M. Jennings ${ }^{2,3}$ - Nikita N. Burke 2,3,4 Michelle Roche $^{3,4}$. \\ Veronica McInerney $^{5}$. Jonathan D. Wren ${ }^{6}$ David P. Finn ${ }^{2,3} \cdot$ Patrick C. McHugh ${ }^{1}$
}

Published online: 1 July 2017

(C) Springer Science+Business Media, LLC 2017

\section{Erratum to: Mol Neurobiol}

DOI 10.1007/s12035-017-0492-8

The original version of this article unfortunately contained mistake. Dr. Veronica McInerney's affilation should be changed to "HRB Clinical Research Facility, National University of Ireland Galway, Galway, Ireland". This is hereby given below.

The online version of the original article can be found at http://dx.doi.org/ $10.1007 / \mathrm{s} 12035-017-0492-8$

Patrick C. McHugh

p.c.mchugh@hud.ac.uk

1 Centre for Biomarker Research and Department of Pharmacy, School of Applied Sciences, University of Huddersfield, Huddersfield HD1 3DH, UK

2 Pharmacology and Therapeutics, National University of Ireland, Galway, Ireland

3 Centre for Pain Research and Galway Neuroscience Centre, National University of Ireland, Galway, Ireland

4 Physiology, School of Medicine, National University of Ireland, Galway, Ireland

5 HRB Clinical Research Facility, National University of Ireland, Galway, Galway, Ireland

6 Arthritis and Clinical Immunology Research Program, Oklahoma Medical Research Foundation, Oklahoma City, OK, USA 